\title{
Intramedullary enterogenous cyst presenting with spastic paraparesis during two consecutive pregnancies: a case report
}

\author{
J W C Reinders, P Wesseling, P H E Hilkens
}

\begin{abstract}
A 35 year old woman presented with two episodes of spastic paraparesis, occurring in the third trimester of two consecutive pregnancies. The neurological symptoms seemed to be caused by an intramedullary cyst in the thoracic spinal cord. The cyst was subtotally removed and histopathologically diagnosed as enterogenous cyst. Other congenital abnormalities were absent. The peculiar timing of the clinical manifestation of an intramedullary cyst has not been described before. An unequivocal explanation for this phenomenon is missing, but several factors related to pregnancy that may play a part are discussed.

(F Neurol Neurosurg Psychiatry 2001;71:528-530)
\end{abstract}

Keywords: intramedullary cyst; enterogenous cyst; spastic paraparesis

Enterogenous cysts are rare lesions of presumed endodermal derivation that can occur in and around the CNS. Other names that have been used for this type of lesion are endodermal, neurenteric, epithelial, bronchogenic, respiratory, and foregut cyst. ${ }^{1}$ Intraspinal enterogenous cysts are generally located in the subarachnoid space anterior to the spinal cord.

Department of Neurology, Rijnstate Hospital, Wagnerlaan 55, 6815 AD Arnhem, The Netherlands J W C Reinders P H E Hilkens

Department of Pathology and Neurology, University Medical Center Nijmegen, P.O. Box $9101,6500 \mathrm{HB}$ Nijmegen, The Netherlands P Wesseling

Correspondence to: Dr PHE Hilkens p.hilkens@worldonline.nl

Received 10 January 2001 and in revised form 20 May 2001

Accepted 3 June 2001 Intramedullary location has been described in only 12 patients. ${ }^{2-17}$

We describe a case of an intramedullary enterogenous cyst with recurrent symptoms presenting in the last trimester of two consecutive pregnancies.

\section{Case report}

A 31 year old woman was examined in the 7 th month of her first pregnancy because she had developed a disturbed sensation in the legs and a disturbance of gait. Headache, nausea, vomiting, visual disturbances, and diplopia were absent. The medical history was remarkable for ulcerative colitis for which she used mesalazine. Neurological examination at that time showed proximal weakness in the left leg and normal strength in the right leg and both arms. Vibration sense was decreased in both legs and the pelvic area. Reflexes were brisk in the arms and very brisk in the legs, with clonic
Achilles tendon reflexes. Plantar reflexes were normal. Laboratory investigation showed mild vitamin B12 deficiency (129 pmol/1, normal range $160-550 \mathrm{pmol} / \mathrm{l}$ ) with normal folic acid. Under the diagnosis of combined degeneration of the spinal cord vitamin B12 supplementation was started. Post partum her symptoms resolved almost completely, only slight paraparesis remained.

At the age of 35 , she was referred to our department of neurology because in the 8th month of her second pregnancy she again developed diminished temperature sensation, paraesthesias in both legs, loss of strength in the legs, and impaired micturition. Post partum these complaints worsened. Neurological examination showed a spastic paraparesis with spastic gait, symmetric hyperreflexia of the legs, and pathological plantar reflexes. Sensation was impaired in both legs with a sensory level at Th10. Thoracic spine MRI showed a large intramedullary cyst at Th8-Th9, filling almost the entire spinal canal (fig 1 ).

A laminectomy with resection of the cyst was performed. As the wall of the cyst adhered to the medullary tissue it could not be completely resected. Histopathologically, the cyst wall consisted of hypocellular connective tissue surrounded by neuroglial tissue. The inside of the wall was covered with one layer of cuboidal to flat cells, sporadically showing a mucin filled cytoplasmatic vacuole (Alcian b blue staining), but without apical cilia. Immunohistochemically, these epitheloid cells showed strong staining for the epithelial markers keratin (fig 2) and epithelial membrane antigen (EMA), but were negative for the glial markers GFAP and S100. The histopathological diagnosis was enterogenous cyst. Postoperatively, the sensory deficit at first deteriorated, but after treatment with corticosteroids this deficit improved in the course of a week and the strength in the legs returned to normal. Two months after operation the only residual deficit was a slight hypaesthesia in the perineal region and lower legs.

\section{Discussion}

Enterogenous cysts are considered congenital anomalies. They are generally located in the mediastinum, the abdominal cavity, and within 

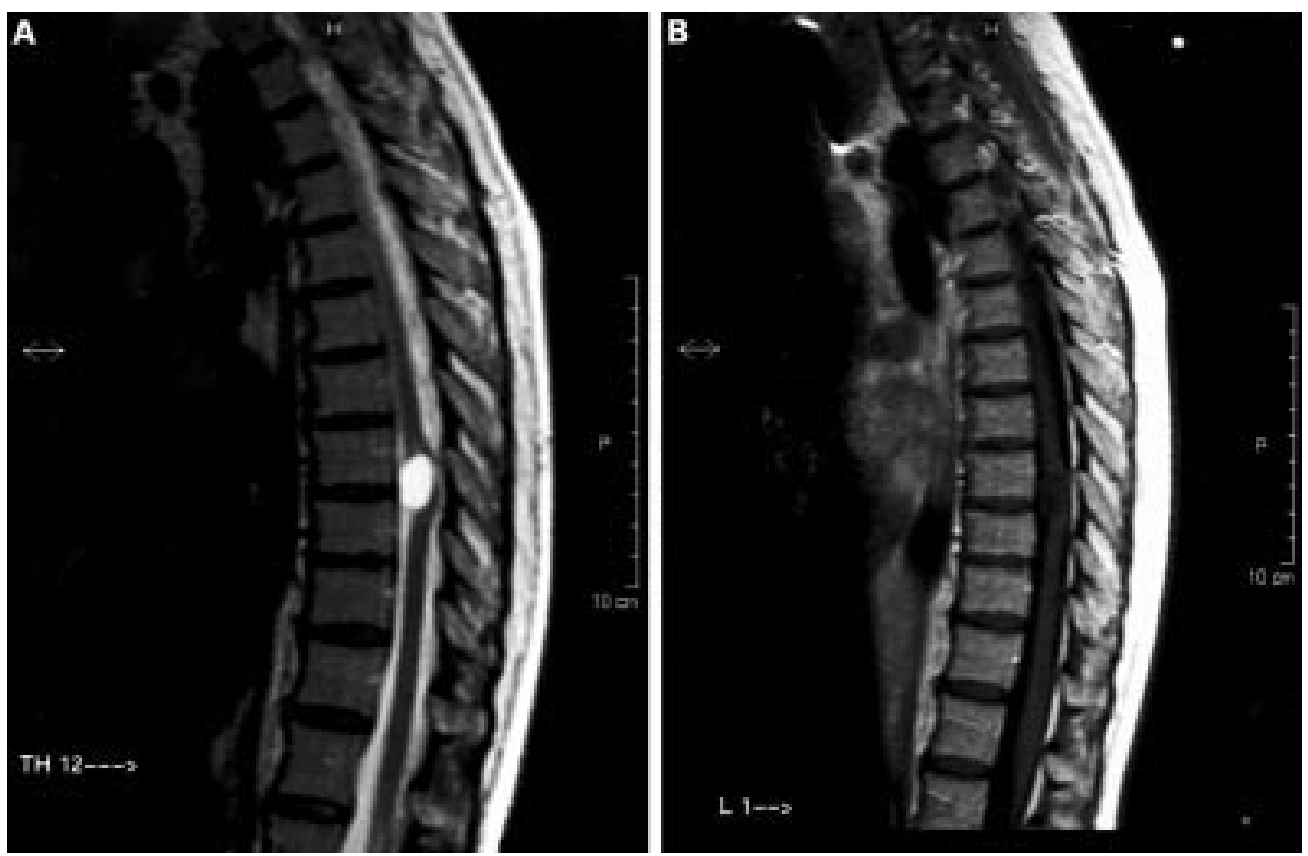

Figure 1 (A) Sagittal T2 weighted MRI of the thoracic spine shows a large cystic lesion at Th8-Th9, filling almost the entire spinal canal. (B) In the gadolinium enhanced sagittal T1 weighted image there is no enhancement of the cyst.

the spinal canal or skull. Intramedullary enterogenous cysts are extremely rare..$^{2-11}$

${ }^{12-17}$ To explain their development various hypotheses have been postulated. ${ }^{235}$ In the hypothesis of the split notochord syndrome, the primary defect is a partial duplication and separation of the notochord (split notochord) with adherence of the ventral endoderm to the dorsal ectoderm and potentially deviant development of surrounding gut, bone, and nervous tissue. Differential growth of the embryo may result in partial obliteration of the fistula. Enterogenous cysts may be associated with congenital anomalies of the skin (for example, hyperpigmentation or hypertrichosis in the midline of the back), and vertebral anomalies. $^{235}$

Histopathologically, the simplest forms of enterogenous cysts are lined by one layer of columnar, ciliated, and goblet cells supported

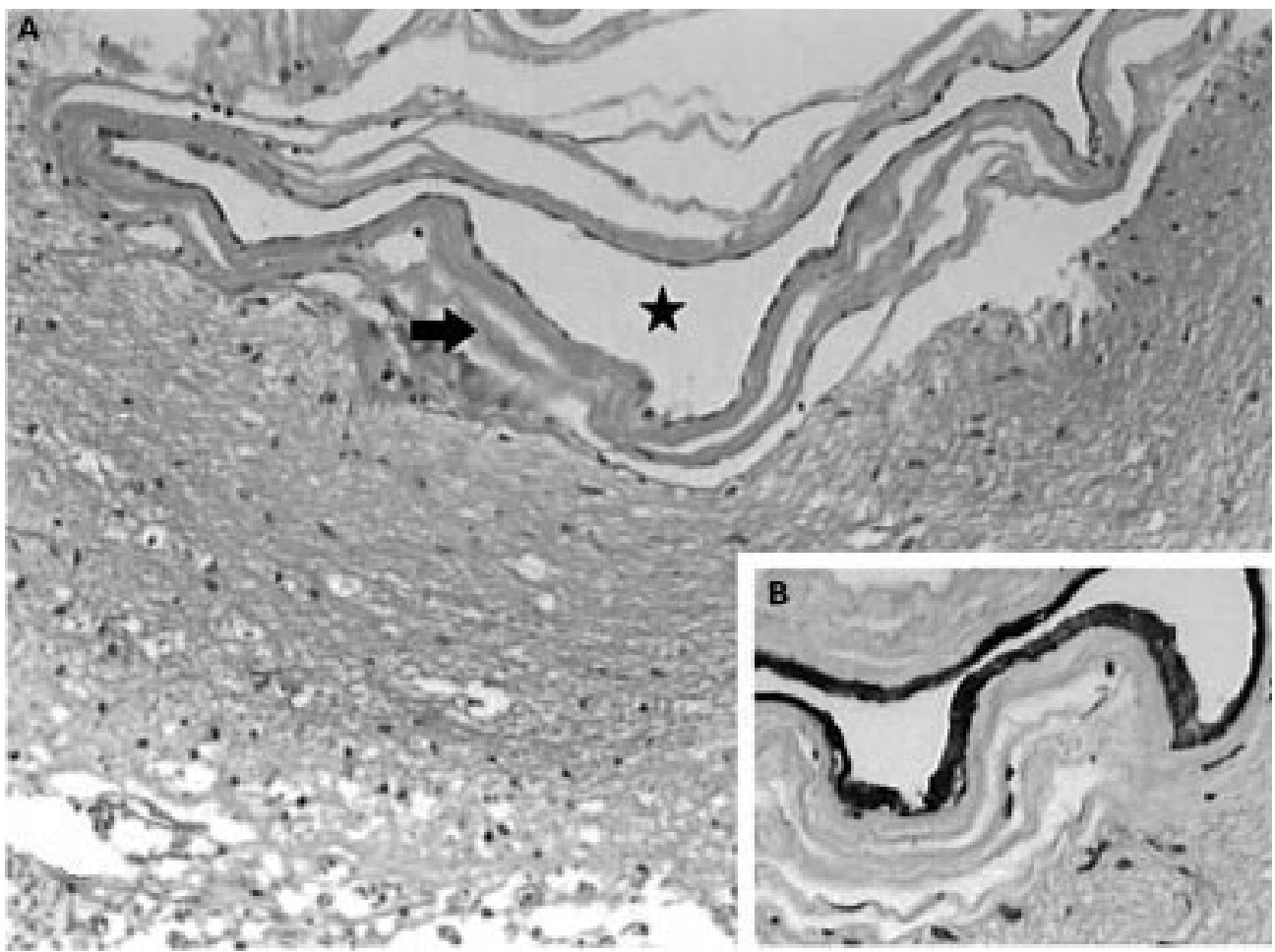

Figure 2 ( $A$ and B) Light microscopy of the intramedullary cyst, showing hypocellular connective tissue in the wall (arrow), surrounded by neuroglial tissue and covered on the inside by one layer of cuboidal to flat cells. The lumen of the cyst is indicated by an asterisk. The inset (B) shows strong immunohistochemical staining of the lining cells for keratin, corroborating the epithelial nature of these cells. (A) haematoxylin and eosin staining; original magnification $\times 100$. (B) Keratin staining; original magnification $\times 250$ ) 
by a layer of connective tissue. More complex types have a similar epithelial lining, but they contain one or more of the additional elements found along the alimentary and respiratory tract.

The clinical signs of intraspinal and intramedullary enterogenous cysts are those of an enlarging intraspinal mass. ${ }^{23}$ The most common clinical course is one of exacerbations and remissions. Possible explanations for this fluctuating clinical course include periodic rupture of the cyst and fluctuations in the rate of production and reabsorption of mucin by the cyst wall.

The optimal treatment for symptomatic intraspinal and intramedullary enterogenous cysts is surgical resection. ${ }^{356}$ Because of adherence of the cyst to the spinal cord complete resection may be impossible. In most cases, however, incomplete resection results in long lasting improvement of symptoms.

An unequivocal explanation for the relation of clinical symptoms and pregnancy in our patient is not available. Theoretically, the following pregnancy related mechanisms might play a part: hormonal influences (causing altered fluid homeostasis), raised intraabdominal pressure (resulting in reduced drainage via the spinal veins, or even in increased intracystic pressure in the case of open connection with the abdominal cavity), altered posture of the spine (with potentially narrowing of the spinal canal and redistribution of blood), or pregnancy related exacerbation of ulcerative colitis. ${ }^{18}$ The reasons for the cyst becoming symptomatic in our patient remain unknown, however.

We thank Dr RH Boerman for revision of the article and Dr FOHW Kesselring for describing the MRI.

1 Rosai J, Dorfman RF. Sinus histiocytosis with massive lymphadenopathy; a newly recognized clinico-pathological entity. Arch Pathol (Chicago) 1969;82:63-70.

2 Foucar E, Rosai J, Dorfman R. Sinus histiocytosis with massive lymphadenopathy (Rosai-Dorfman disease): review of the entity. Semin Diagn Pathol 1990;7:19-73.

$3 \mathrm{Kim} \mathrm{M,} \mathrm{Provias} \mathrm{J,} \mathrm{Bernstein} \mathrm{M.} \mathrm{Rosai-Dorfman} \mathrm{disease}$ mimicking multiple meningioma: case report. Neurosurgery 1995;36:1185-7.

4 ShaverEG, Rebsamen SL, Yachnis AT, et al. Isolated extranodal intracranial sinus histiocytosis in a 5 year old boy: case report. F Neurosurg 1993;79:769-73.

5 Lopez P, Estes ML. Immunohistochemical characterization of the histiocytes in sinus histiocytosis with massive lymphadenopathy: analysis of an extranodal case. Human Pathol 1989;20:711-15.

6 Foucar E, Rosai J, Dorfman RF. The neurologic manifestations of sinus histiocytosis with massive lymphadenopathy. Neurology 1982;32:365-71.

7 Song SK, Schwartz IS, Strauchen JA, et al. Meningeal nodules with features of extranodal sinus histiocytosis with massive lymphadenopathy. Am F Surg Pathol 1989;13:40612.
8 Wenig BM, Abbondanzo SL, Childers EL, et al. Extranodal sinus histiocytosis with massive lymphadenopathy RosaiDorfman disease) of the head and neck. Human Pathol 1993;24:483-92

9 Bhattacharjee $\mathrm{MB}$, Wroe SJ, Harding $\mathrm{BN}$, et al. Sinus histiocytosis with massive lymphadenopathy: isolated suprasellar involvement. F Neurol Neurosurg Psychiatry 1992;55:156-8.

10 Woodcock RJ, Mandell JW, Lipper MH. Sinus histiocytosis (Rosai-Dorfman disease) of the suprasellar region: MR imaging findings: a case report. Radiology 1999;213:80810.

11 Resnick DK, Johnson BL, Lovely TJ. Rosai-Dorfman disease presenting with multiple orbital and intracranial masses. Acta Neuropathol 1996;91:554-7.

12 Olsen EA, Crawford JR, Vollmer RT. Sinus histiocytosis with massive lymphadenopathy and unusual extranodal manifestations. Am Pathol Lab Med 1988;18:1322-32.

13 Friedman MJ, Rossoff LJ, Aftalion B, et al. Sinus histiocytosis presenting as a mediastinal mass. Chest 1984;86:266-7.

14 Udono H, Fukuyama K, Okamoto $\mathrm{H}$, et al. Rosai-Dorfman disease presenting multiple intracranial lesions with unique findings on magnetic resonance imaging: case report. 7 findings on magnetic resona

15 Katz DS, Poe LB, Corona RJ. Sinus histiocytosis with massive lymphadenopathy: a case of simultaneous upper respiratory-tract and CNS disease without lymphadenopathy. Am f Neuroradiol 1993;14:219-22.

$16 \mathrm{Ng} \mathrm{HK}$, Poon WS. Sinus histiocytosis with massive lymphadenopathy localized to the sella. $\mathrm{Br} f \mathrm{f}$ Neurosurg 1995;9:551-5.

17 Clark WC, Berry, AD. Extranodal sinus histiocytosis with massive lymphadenopathy: isolated central nervous system involvement mimicking meningioma. South Med f 1996;89: 621-3.

18 Panicker NK, Sabhikhi AK, Rai R. Rosai-Dorfman disease presenting as a meningioma. Indian $\mathcal{F}$ Cancer 1996;33:192-

19 Deodhare SS, Ang LC, Bilbao JM. Isolated intracranial involvement in Rosai-Dorfman disease: a report of two cases and review of the literature. Arch Pathol Lab Med 1998;122:161-5.

20 Morandi X, Godey B, Riffaud L, et al. Isolated RosaiDorfman disease of the fourth ventricle: case illustration. $\mathcal{F}$ Neurosurg 2000;92:890.

21 Asai A, Matsutani M, Kohno T, et al. Leptomeningeal and orbital benign lymphophagocytic histiocytosis: case-report. f Neurosurg 1988;69:610-12.

22 Carey MP, Case CP. Sinus histiocytosis with massive lymphadenopathy presenting as a meningioma. Neuropathol Appl Neurobiol 1987;13:391-8.

23 Friendly DS, Font RL, Rao NA. Orbital involvement in sinus histiocytosis. Arch Ophthalmol 1977;95:2006-11.

24 Mir R, Aftalion B, Kahn LB. Sinus histiocytosis with massive lymphadenopathy and unusual extranodal manifestations. Arch Pathol Lab Med 1985;109:867-70.

25 Mirra SS, Tindall SC, Check IJ, et al. Inflammatory meningeal masses of unexplained origin: an ultrastructural and immunological study. F Neuropathol Exp Neurol 1983;42: 453-68.

26 Trudel M. Dural involvement in sinus histiocytosis with massive lymphadenopathy: case report. F Neurosurg 1984; 60:850-2.

27 Gaetani P, Tancioni T, Dirocco M, et al. Isolated cerebellar involvement in Rosai-Dorfman disease: case report. Neurosurgery 2000;46:479-81.

28 Huang HY, Huang CC, Lui CC, et al. Isolated intracranial Rosai-Dorfman disease: case report and literature review. Pathol Int 1998;48:396-402.

29 Kudesia S, Shankar SK, Das S, et al. Intracranial plasma cell granuloma: a report of four cases. Neurol India 1998;46: 142-6.

30 Stromberg JS, Wang AM, Huang TE, et al. Langerhans cell histiocytosis involving the sphenoid sinus and superior orbital fissure. Am ₹ Neuroradiol 1995;16:964-7.

31 Destombes P. Adénites avec surcharge lipidique, de l'enfant ou de l'adulte jeune, observées aux Antilles et au Mali. Bull Soc Pathol Exot 1965;6:1169-75. 